\title{
Study on Positioning High Dynamic Distributed Platforms Based on LFM Signal
}

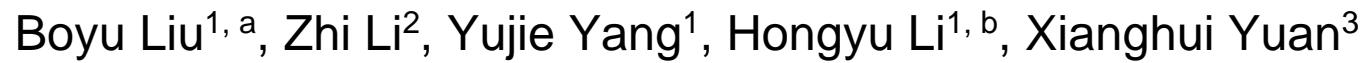 \\ ${ }^{1}$ Beijing Institute of Radio Metrology and Measurement, Beijing 100854, China; \\ ${ }^{2}$ Corporation Science and Technology council of Defense Technology Academy of China Aerospace \\ Science \& Industry Corporation, Beijing 100854, China; \\ ${ }^{3}$ School of Electronic Engineering, Naval University of Engineering, Wuhan, Hubei 430033, China \\ a358756369@qq.com, blihongyu203@sina.cn
}

\begin{abstract}
Proposed a positioning method for high dynamic distributed platforms combined two-way microwave ranging with short baseline interferometer angle measurement. Effectively suppressed Doppler interference while ranging based on the frequency and time characteristics of broadband Positive and negative slope of the LFM (linear frequency modulation) signal. Also proposed precise angle measurement method with ambiguity resolution by frequency measurement. Finally, analyzed and experimental verified the validity and accuracy of the positioning method.
\end{abstract}

Keywords: High dynamic distributed platform; Short-line interferometer; Platforms positioning; Positive and negative slope linear frequency modulation (LFM) signal.

\section{Introduction}

High-precision positioning technology in satellite launching, guidance, navigation, communications, transportation and other fields has a pivotal position. In some specific areas, the positioning accuracy has become one of the key factors to enhance the performance of distributed work system devices. GPS (Global Positioning System) could achieve high accuracy positioning, but susceptible to specific interference, so the independent relative positioning between high dynamic distributed platforms has become an urgent problem.

Static distributed systems usually use multi-platforms TDOA technology. By measuring the distances between the target and base platforms, we could achieve high precise position combining the position information of base platforms. But high dynamic distributed systems usually consist of small equipments and high dynamic movements, the distance between the receivers, power consumption and so on all have strict requirements, so the TDOA technology will achieve low positioning accuracy. What's more, The Doppler effect also causes great interference to the relative positioning between platforms.

\section{Positioning principle introduction}

Collaborative work platforms usually use active distributed collaborative approach to achieve mutual positioning, larger distributed devices typically realize positioning based on TOA(time of arrival) method, we could get at least three spheres by measuring the time from the target station to the reference stations, then get the target position based on the intersection coordinates of the spherical. However, the high dynamic distributed devices' volume is strictly limited, they could only target mutually with a single platform by setting multiple antennas instead of base stations, the TOA positioning method would lead to large error in this situation [2-4]. With high consistency of each antenna channels, the TDOA (time difference of arrival) positioning method would be a better choice. The main platform achieves positioning by calculating the intersection of multiple hyperboloid based on the time difference between the adjacent antennas.

To minimize the complexity of the equipments and the algorithms, a new positioning method combined the two dimensional angle measurement with ranging is proposed. The main platform arranges three antennas as two short baselines by an ' $\mathrm{L}_{-}$form. By the method of two-way microwave 
ranging and phase interferometer angle measurement, we can get the vice platform position at the main platform antenna coordinate system.

The antenna coordinate system of main platform is constituted by three vertical distributed antennas. As is shown in figure 1.

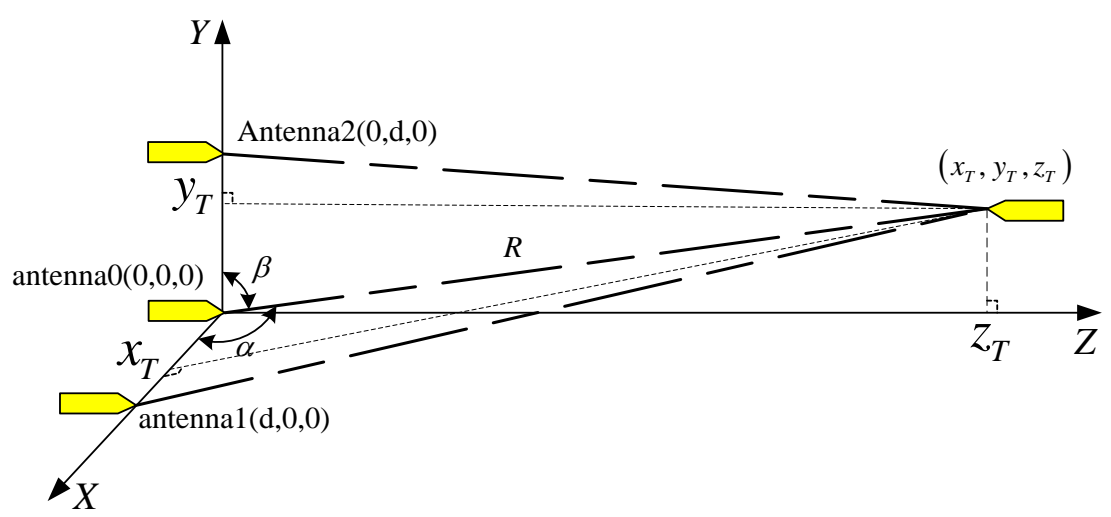

Fig.1 Geometrical relationship of antennas

Baseline length is $\mathrm{d}$, the coordinates of antenna1 and antenna 2 in the main platform antenna coordinate system are $(d, 0,0)$ and $(0, d, 0)$, the vice platform's coordinate is $\left(x_{T}, y_{T}, z_{T}\right)$, then its expression is.

$$
\left\{\begin{array}{l}
x_{T}=\left(R+e_{R}\right) \cos \left(\alpha+e_{\alpha}\right) \\
y_{T}=\left(R+e_{R}\right) \cos \left(\beta+e_{\beta}\right) \\
z_{T}=\left(R+e_{R}\right) \sqrt{1-\cos ^{2}\left(\alpha+e_{\alpha}\right)-\cos ^{2}\left(\beta+e_{\beta}\right)}
\end{array}\right.
$$

Where $\mathrm{R}$ is the distance between the main platform antenna 0 and the vice platform, $\alpha, \beta$ is the angle with axis $\mathrm{X}$ and axis $\mathrm{Y} . e_{R}, e_{\alpha}, e_{\beta}$ is the distance and angle measurement noise with zero mean, they are all zero in the ideal case. Assumed the parameter estimation variances are $\sigma_{R}{ }^{2}, \sigma_{\alpha}{ }^{2}, \sigma_{\beta}{ }^{2}$, and $\sigma_{\alpha}{ }^{2}=\sigma_{\beta}{ }^{2}$.The distance $\mathrm{R}$ is obtained by two-way microwave method, the angle $\alpha, \beta$ are obtained by the short line interferometer.

\section{Ranging Model Based on Positive and Negative Slope LFM Signal}

\subsection{Principle of Suppressing the Doppler Effect.}

The positive and negative slope LFM signal could suppress the influence of Doppler and poor frequency stability, and reduce the pressure of IF sampling and back-end signal processing [5]. The principle of suppressing the Doppler interference works as follows:

When the platforms are in a stationary state, the relationship between time and frequency of the dechirped positive and negative slope of the LFM signal is shown in figure 2.

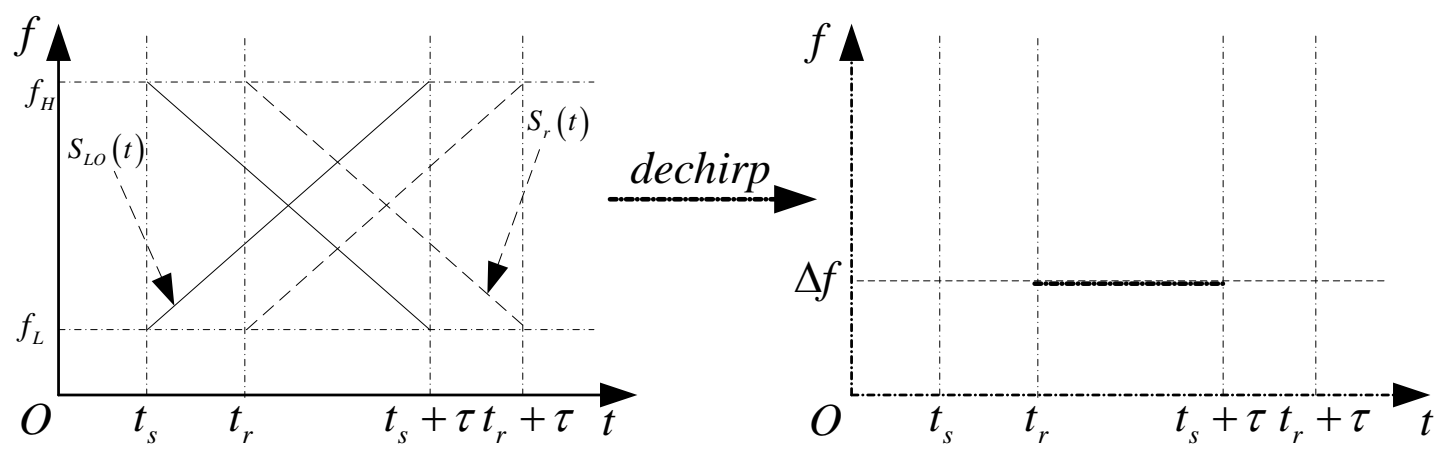

Fig.2 Dechrip processing between static platforms

The IF frequency obtained by mixing the local oscillation signal and the echo signal is $\Delta f$, due to the positive and negative slope LFM signals are transmitted simultaneously, It will also generate a 
double modulated slope LFM signal when the positive and negative slope LO signal mixed with opposite slope echo signal, which is referred to as the difference-frequency interference signals. The frequency of the difference-frequency interference signal can be controlled in certain range. Its duration time is short in the filter band after filtering process, and it's energy is negligible compared to the IF signal's, so the difference-frequency interference signal will make no influence on the measurement result.

When the platforms are in high dynamic state, the relationship between time and frequency of the dechirped positive and negative slope of the LFM signal is shown in figure 3.

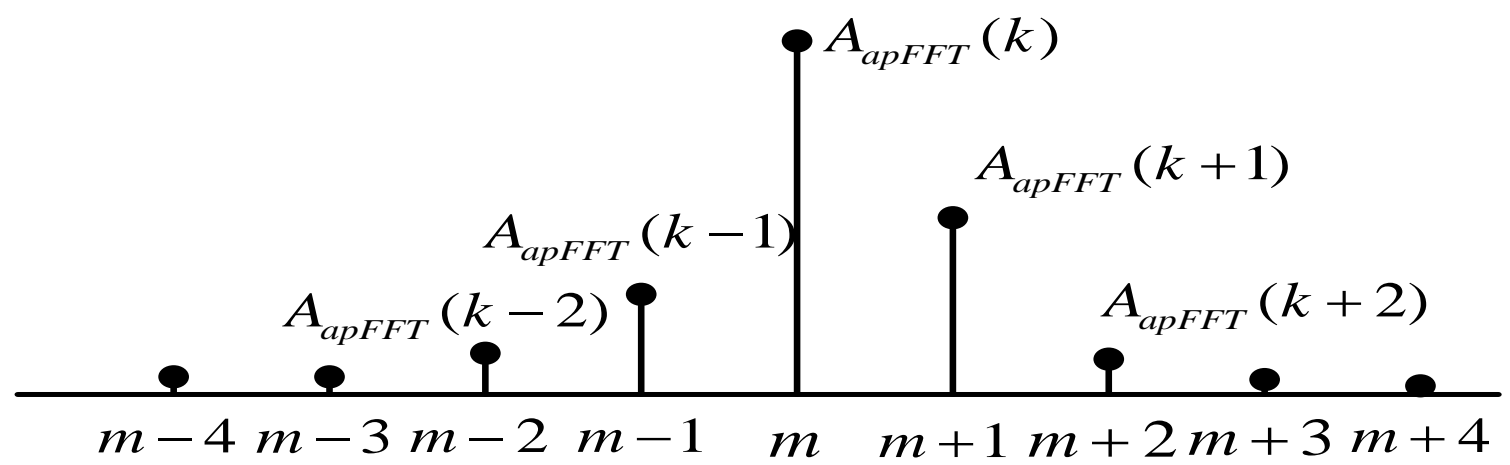

Fig.3 Dechrip processing between moving platforms

The blue line in Figure 3 is the Doppler RF signal generated by the original RF signal and the Doppler signal. After mixing with the LO signal whose parameter is the same as the original RF signal, the frequency of two IF signals are $\Delta f+f_{d}$ and $\Delta f-f_{d}$. By averaging the two frequency or making a direct weight mean estimate with two adjacent peaks in the spectrum, the $f_{d}$ can be counteractive, then we will get $\Delta f$.

The reference frequency difference between different moving platforms effects familiar to the Doppler interference, it could also be suppressed by the dechirp of positive and negative slope LFM signal. Frequency difference is considered as a constant in a short time. The frequency residiuals are negligible after average process. So the dechirp process could not only counteract most of the Doppler Effect and the ref-frequency difference interference but also reduce the pressure on signal process section.

\subsection{Ranging Model based on Two-way Microwave Technology.}

LFM signal combined with the two-way microwave technology could realize accurate ranging between high dynamic platforms. Assuming the distance between primary platform and vice platform is $\mathrm{R}$, the FM slope of the LFM signal is K, then the ranging model is shown in Figure 4.

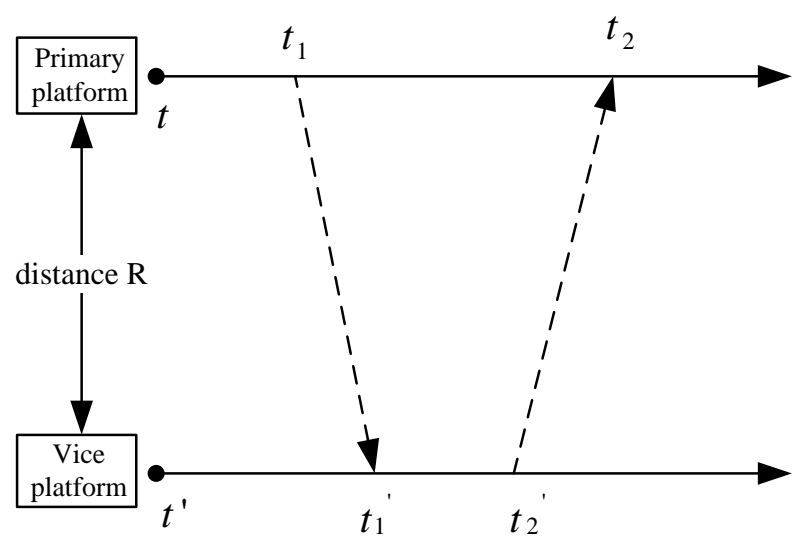

Fig. 4 Timing sequence of time synchronization and ranging

As shown in Figure $4, t_{1}$ is the primary platform moment that the primary platform sends the measured signal, $t_{1}{ }^{\prime}$ is the vice platform moment that the measured signal arrives the vice platform, $t_{2}{ }^{\prime}$ is the vice platform moment that the vice platform sends the measured signal, $t_{2}$ is the primary 
platform moment that the measured signal arrives the primary platform. Since the two platforms are not synchronized, the time difference sets $\delta_{t}$, then there is a formula.

$$
\begin{aligned}
& t_{1}+\text { Delay }_{P S}+\frac{R}{C}+\text { Delay }_{\mathrm{VR}}=t_{1}{ }^{\prime}+\delta_{t}=t_{L O}{ }^{\prime}-\frac{f_{1}{ }^{\prime}}{K}+\delta_{t} \\
& t_{2}{ }^{\prime}+\text { Delay }_{\mathrm{VS}}+\frac{R}{C}+\text { Delay }_{P R}=t_{2}-\delta_{t}=t_{\mathrm{LO}}-\frac{f_{2}}{K}-\delta_{t}
\end{aligned}
$$

Where Delay $_{X X}$ is the system delay, $t_{L O}, t_{L O}{ }^{\prime}$ is the LO signal generating moment, $f_{1}{ }^{\prime}, f_{2}$ is the frequency dechirped of the primary and vice platform.

Suppose $\Delta t_{a}=$ Delay $_{P S}+$ Delay $_{\mathrm{VR}}, \Delta t_{b}=$ Delay $_{\mathrm{VS}}+$ Delay $_{P R}$, then the results are.

$$
\begin{aligned}
& \delta_{t}=\frac{\left[t_{1}-\left(t_{L O}{ }^{\prime}-\frac{f_{1}{ }^{\prime}}{K}\right)\right]+\left[\left(t_{\mathrm{LO}}-\frac{f_{2}}{K}\right)-t_{2}{ }^{\prime}\right]}{2}+\frac{\Delta t_{a}-\Delta t_{b}}{2} \\
& R=\left[\frac{\left[\left[\left(t_{\mathrm{LO}}-\frac{f_{2}}{K}\right)-t_{1}\right]-\left[t_{2}{ }^{\prime}-\left(t_{L O}{ }^{\prime}-\frac{f_{1}^{\prime}}{K}\right)\right]\right.}{2}-\frac{\Delta t_{a}+\Delta t_{b}}{2}\right] \cdot c
\end{aligned}
$$

$t_{1}, t_{\mathrm{LO}}, t_{2}{ }^{\prime}, t_{L O}{ }^{\prime}$ are obtained by system timing, the accuracy can be better than ns level, $\Delta t_{a}, \Delta t_{b}$ is inherent system delay bias and could be eliminated by calibration.

\section{Angle Measurement Model based on Short Baseline Interferometer}

\subsection{Traditional Interferometer Angle Measuring Technology.}

Phase interferometer angle measurement technique makes use of the presence of a phase difference while echo signal reach different antennas, thereby calculating the target direction angle. Compared with other methods, phase interferometer system has the advantages of high sensitivity, high accuracy, fast speed, and the ability of anti-wave front distortion.

Traditional interferometer system exist two important questions [6-8]:First, most interferometers work in passive reception mode, although there are certain advantages in electronic warfare, but this mode is at the expense of comprehensive grasp of the target emission waveform feature parameters. Such systems are difficult to design, and the accuracy and response speed is not high. The application scope is usually of strict limitation, especially for a certain specific objectives. The high-precision interferometer systems always work in active or semi-active coherent mode, under the premise of controlling more signal parameters these systems could take the initiative to send measure signal with excessive reliance on the target parameters. The new interferometer method discussed in this article is semi-active intervention system. Second, the interferometer exists the contradiction of the angle resolution and phase difference ambiguity. Double platform interferometer, for example, if the base line is shorter than half the wavelength, the angle could be obtained directly without the ambiguity, but the angle resolution is limited at this time. The resolution could be improved by increasing the length of the base line, but if the base line is longer than half of the carrier wavelength, the phase difference ambiguity should be sloved first to get the angle of the target.

The program solve the phase ambiguity by measuring frequency base on the positive and negative slope broadband LFM Signal. By dechirping LFM signal, the frequency and phase information associated to the TDOA are obtained, and high-precision angle measurement based on short baseline is achieved [9]

\subsection{Introduction of Short baseline interferometer system}

Baseline length of the interferometer is $d$, the angel of the vice platform to the primary platform is measured by calculating the path difference to two receiving antennas. As shown in Figure 5, when the two platform are far apart from each other $\min \left(R_{1}, R_{2}\right) \square d$, the relationship between the wave path difference $\Delta R$, direction angle $\theta$ and base line length $d$ could be considered as:

$$
\Delta \mathrm{R}=R_{2}-R_{1}=\mathrm{d} \cdot \sin \theta .
$$



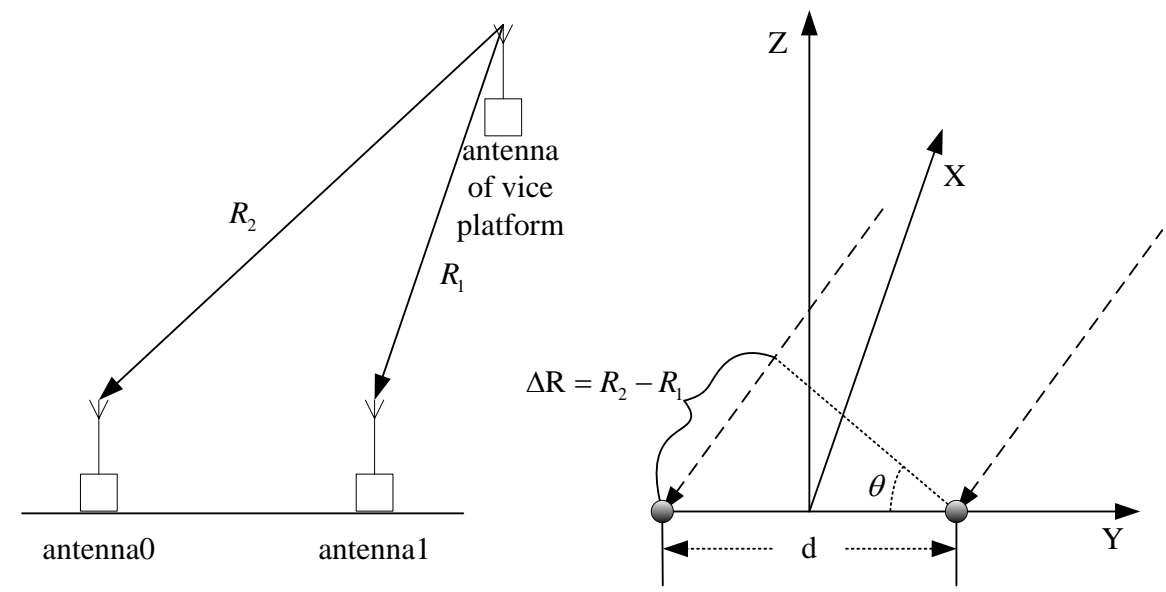

Fig. 5 Schematic diagram of short baseline interferometer

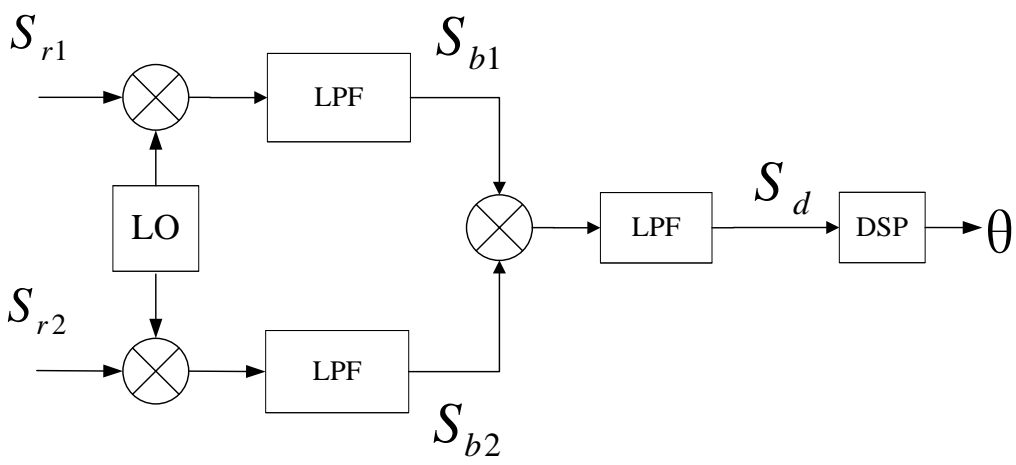

Fig.6 Block diagram of DF system

The angle measurement system block diagram is shown in Figure 6. $S_{r 1}, S_{r 2}$ Are the signals received by the antennas on the primary platform? By mixing with the local oscillation signal, the signals received would turn to low frequency continuous signal $S_{b 1}$ and $S_{b 2}$, which are related to the distance. By mixing and filtering, the angle measurement would be realized after the ambiguity is solved.

\subsection{Ambiguity Resolution Principle Analysis.}

To facilitate the analysis, the real signal is replaced by complex signal. For a single measured target [10-12]:

$$
\begin{aligned}
& S_{r 1}(t)=A_{2} e^{j\left[2 \pi f_{0}\left(t-t_{1}\right)+\pi k\left(t-t_{1}\right)^{2}\right]}+n_{r 1}(t) \\
& S_{r 2}(t)=A_{2} e^{j\left[2 \pi f_{0}\left(t-t_{2}\right)+\pi k\left(t-t_{2}\right)^{2}\right]}+n_{r 2}(t) \\
& S_{L}(t)=e^{j\left[2 \pi f_{0}\left(t-t_{d}\right)+\pi k\left(t-t_{d}\right)^{2}\right]}
\end{aligned}
$$

Where $n_{r 1}(t)$ and $n_{r 2}(t)$ are noise mixed in the signal components, $\mathrm{k}$ is the modulate slope of the linear LFM signal and $k=\frac{B}{T}$, then we could get

$$
\begin{aligned}
& S_{b 1}(t)=A_{1} \cdot e^{j\left(2 \cdot \pi \cdot f_{b 1} \cdot t+\varphi_{b 1}\right)}+n_{b 1}(t), t_{d 1} \leq t \leq t_{1}+T \\
& S_{b 2}(t)=A_{1} \cdot e^{j\left(2 \cdot \pi \cdot f_{b 2} \cdot t+\varphi_{b 2}\right)}+n_{b 2}(t), t_{d 2} \leq t \leq t_{2}+T
\end{aligned}
$$

Where

$$
\begin{aligned}
& f_{b 1}=k\left(t_{d}-t_{1}\right) \\
& f_{b 2}=k\left(t_{d}-t_{2}\right) \\
& \varphi_{b 1}=2 \pi f_{0}\left(t_{d}-t_{1}\right)+\pi k\left(t_{1}^{2}-t_{d}^{2}\right) \\
& \varphi_{b 2}=2 \pi f_{0}\left(t_{d}-t_{2}\right)+\pi k\left(t_{2}^{2}-t_{d}^{2}\right)
\end{aligned}
$$


After dechirp processing, noise bandwidth of the receiver can reduce to $1 / T$ from $B$, which would improve the SNR effectively. In order to eliminate the impact of leading-edge jitter, we could mix $S_{b 1}$ and $S_{b 2}$ then filter the high frequency component. The low frequency pulse continuous signal $S_{d}$ related to the direction angle $\theta$ would be obtained. The analytic form shown below.

$$
S_{d}(t)=A_{d} e^{j\left(2 \pi f_{d} t+\phi_{d}\right)}+n_{d}(t), t_{d 2} \leq t \leq t_{1}+T
$$

The relationship between frequency, phase and direction angle is as follows

$$
\begin{aligned}
& f_{d}=f_{b 2}-f_{b 1}=k\left(\frac{\Delta R}{c}\right)=\frac{k d}{c} \cdot \sin \theta \\
& \varphi_{d}=\varphi_{b 2}-\varphi_{b 1}=2 \pi f_{0}\left(t_{1}-t_{2}\right)-\pi k\left(t_{1}-t_{2}\right)\left(t_{1}+t_{2}\right)=\pi\left[2 f_{0}-k\left(t_{1}+t_{2}\right)\right] \sin \theta d / c
\end{aligned}
$$

Thus, the angle measurement could be realized by measuring $f_{d}$ or $\varphi_{d}$, but there are big differences between the two methods, generally speaking, measuring the angle with $\varphi_{d}$ would lead to higher accuracy.

From the above equations, the frequency variation corresponding to phase variation is as follows.

$$
f_{\varphi}=\frac{\varphi_{d}}{2 \pi\left(\frac{f_{0}}{k}-\frac{R}{c}\right)}
$$

Where $\mathrm{R}$ is the distance from vice platform to primary platform, $\mathrm{d}$ is the base line length.

According the Cramer-Rao inequality of unbiased vector estimation, the low estimate limit of frequency and phase are as follows.

$$
\begin{aligned}
& \delta_{\hat{f}}=\frac{1}{2 \pi} \sqrt{\frac{12 f_{s}^{2}}{r\left(N^{2}-1\right) N}} \approx \frac{f_{s}}{\pi N} \sqrt{\frac{3}{r N}}=\frac{1}{\pi \tau} \sqrt{\frac{3}{r N}} \\
& \delta_{\hat{\phi}}=\sqrt{\frac{2(2 N-1)}{N(N+1) r}} \approx 2 \sqrt{\frac{1}{r N}}
\end{aligned}
$$

Where $\tau$ is the sample time, $\mathrm{r}$ is $\mathrm{SNR}$ and $\mathrm{N}$ is data points.

According to the formulas above, the frequency variance corresponds to $\delta_{\varphi}$ be.

$$
f_{\delta \varphi}=\frac{2 \sqrt{\frac{1}{r N}}}{2 \pi\left(\frac{f_{0}}{k}-\frac{R}{c}\right)}
$$

The frequency variance corresponds to phase $2 \pi$ variance is

$$
f_{2 \pi}=\frac{1}{\left(f_{0} / k-R / c\right)}
$$

When $f_{\delta \varphi}<\delta f$, the angle measurement accuracy based on phase is higher than that based on frequency, but due to the phase cyclical characteristic, the measurement result exists ambiguity which effects the angle measurement range. Angle measurement based on frequency with the same accuracy need higher frequency measurement accuracy, which will require more to the SNR, sample rate, duration, etc. and it will bring great pressure on the system parameters and data processing. Therefore, the use of two separate methods have certain drawbacks.

$$
\text { When }\left\{\begin{array}{l}
\frac{f_{\delta \varphi}}{\delta f}=\frac{B}{\sqrt{3}\left(f_{0}-\frac{R k}{c}\right)}<1 \\
\frac{f_{2 \pi}}{\delta f}=\sqrt{\frac{r N}{3}} \frac{\pi \tau}{\left(f_{0} / k-R / c\right)}>1
\end{array}, \text { angle measurement accuracy based on } \varphi_{d}\right. \text { is higher than that }
$$

based on $f_{d}$.and the phase ambiguity could be solved by frequency measurement. The combination of two methods keep the angle measurement accuracy and reduce the requirement on frequency measurement accuracy at the same time. Thus the high-precision direction angle measurement based on single base line and wide measurement range is achieved. 


\section{Accuracy Analysis and Experimental Evaluation}

\subsection{Ranging Accuracy Analysis.}

According to the ranging formula, the error is defined as

$$
\delta(R)=c \cdot \delta\left(\frac{\left(t_{2}-t_{1}\right)+\left(t_{2}{ }^{\prime}-t_{1}{ }^{\prime}\right)}{2}\right)+c \cdot \delta\left(\frac{\Delta t_{a}-\Delta t_{b}}{2}\right)
$$

While $t_{1}{ }^{\prime}=t_{L O}{ }^{\prime}-\Delta t_{1}, t_{2}=t_{\mathrm{LO}}-\Delta t_{2}, f_{1}{ }^{\prime}=K \times \Delta t_{1}, f_{2}{ }^{\prime}=K \times \Delta t_{2}$, the error formula can be derived as

$$
\delta\left(\delta_{t}\right)=\delta\left(\frac{\left(t_{L O}-t_{1}\right)-\left(t_{2}{ }^{\prime}-t_{\mathrm{LO}}\right)}{2}\right)-\delta\left(\frac{f_{1}{ }^{\prime}+f_{2}{ }^{\prime}}{2 K}\right)-\delta\left(\frac{\Delta t_{a}-\Delta t_{b}}{2}\right)
$$

The ranging error could be divided into three parts:

1. The error caused by system clock

Since $t_{1}, t_{\mathrm{LO}}, t_{2}{ }^{\prime}, t_{L O}{ }^{\prime}$ are obtained by system clock timing, the instability of system reference time will impact the time synchronization.

2. The error caused by frequency estimation

Frequency estimation error is mainly consist by estimate error caused by frequency measurement algorithm. The Cramer-Rao bound of frequency estimation could be considered as the minimum frequency estimation error.

3 . The error caused by inconsistency of transceiver channel

$\Delta t_{a}, \Delta t_{b}$ In formula (24) are equipment system delays, since the inconsistency of transfer channel and receive channel actually exist, the inconsistency will surely cause distance measuring error.

\subsection{Positioning Accuracy Analysis}

Typically positioning accuracy is described by the GDOP (geometrical dilution of precision) [13], whose expression is.

$$
G D O P=\sqrt{\operatorname{tr}(P)}
$$

Where $\operatorname{tr}()$ means matrix trace, $\mathbf{P}$ presents the positioning error covariance matrix.

According to the positioning process, the coordinate equations could be obtained.

$$
\left\{\begin{array}{l}
x_{T}=R \cos \alpha \\
y_{T}=R \cos \beta \\
z_{T}=R \sqrt{1-\cos ^{2} \alpha-\cos ^{2} \beta}
\end{array}\right.
$$

Intergrating and organizing the equations.

$d x=C d v+d e$

Where $d x=\left[d x_{T}, d y_{T}, d z_{T}\right]^{T}$ is the coordinate error matrix; $d v=[d R, d \alpha, d \beta]^{T}$ is the distance and angle measurement error matrix; $d e=\left[d e_{1}, d e_{2}, d e_{3}\right]^{T}$ is the measurement noise matrix.

$$
d C=\left(\begin{array}{ccc}
\cos \alpha & -R \sin \alpha & 0 \\
\cos \beta & 0 & -R \sin \beta \\
\sqrt{1-\cos ^{2} \alpha-\cos ^{2} \beta} & \frac{2 \cos \alpha \sin \alpha}{\sqrt{1-\cos ^{2} \alpha-\cos ^{2} \beta}} & \frac{2 \cos \beta \sin \beta}{\sqrt{1-\cos ^{2} \alpha-\cos ^{2} \beta}}
\end{array}\right)
$$

Since the ground clutter, multipath interference and equipment vibration will all effect the distance and angle measurement, the distance measuring noise and angle measuring noise cannot ignored. Therefore the least squares method is required to get $d x$. The target positioning error covariance matrix is

$$
P=E\left[d x d x^{T}\right]=E\left[C d v d v^{T} C^{T}\right]=C P v C^{T}
$$

Where $P v=d v d v^{T}$. The positioning GDOP of vice platform is

$$
G D O P=\sqrt{d x_{T}{ }^{2}+d y_{T}{ }^{2}+d z_{T}{ }^{2}}=\sqrt{\operatorname{tr}(P)}
$$




\subsection{Experimental evaluation.}

The baseline length of primary platform is $0.05 \mathrm{~m}$, the distance between two platforms is $100 \mathrm{~m}$, the signal received by antennas of primary platform could be considered as parallel wave at this time. The frequency band of positive and negative slope LFM signals is Ka and the sweep slope and sweep bandwidth are set at reasonable range. Then comparing the measurement result and actually result of the movement locus of the vice platform.
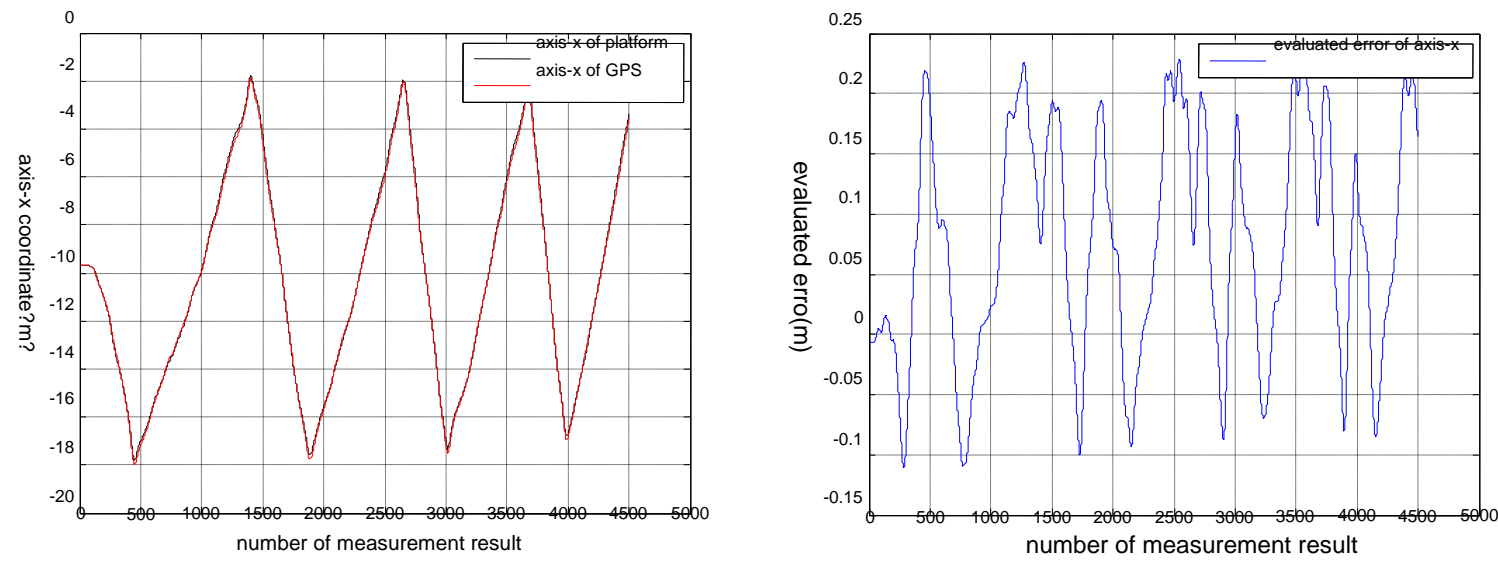

(a)Measurement result and evaluation error of axis- $\mathrm{X}$
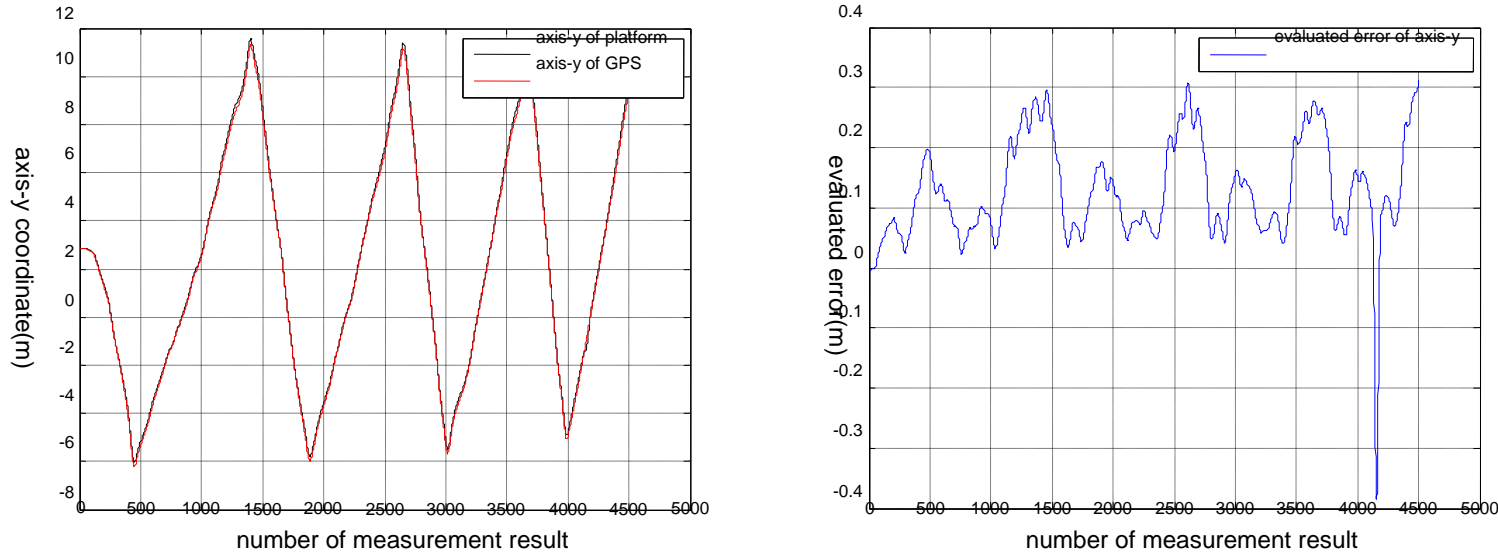

(b)Measurement result and evaluation error of axis-y
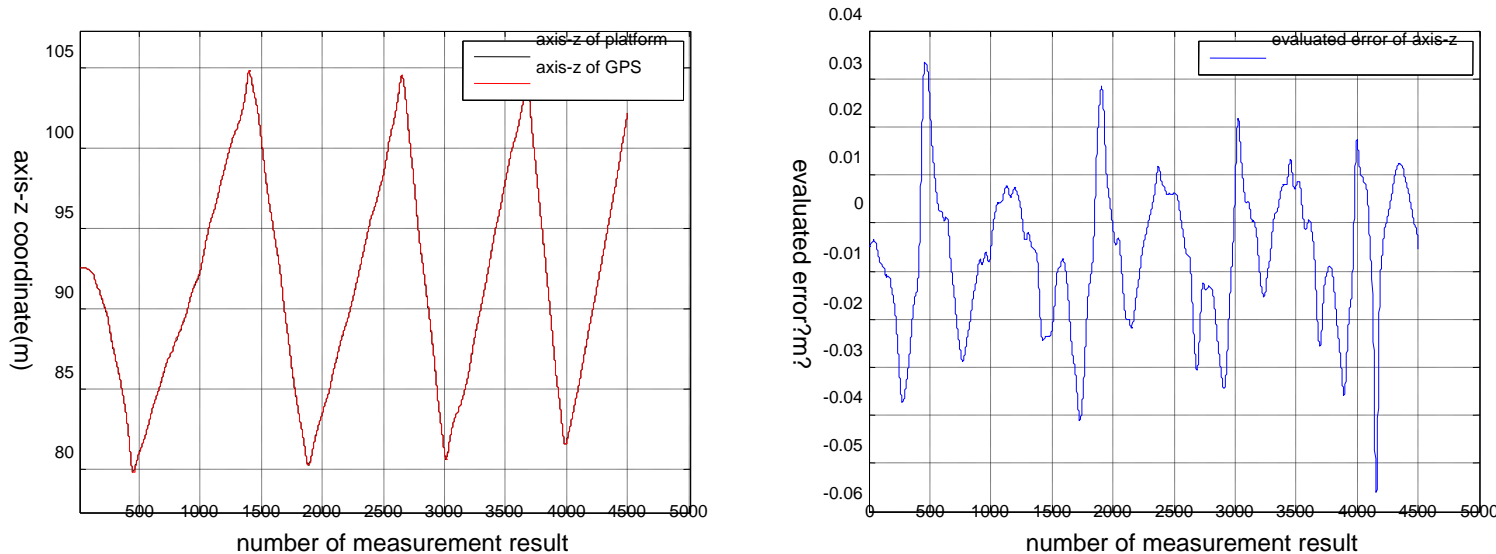

(c)Measurement result and evaluation error of axis-Z

Fig.7 Measurement results and evaluation errors

As is shown in figure 7 , trajectory of vice platform go through the plane perpendicular to the ground contains the z-axis. The amplitude of the received signal is maximum while facing the antenna directly according to the antenna gain characteristics. The distance and angle measurement error is relatively small at this moment. Measurement error is consistent with the theoretical analysis. 
Through the analysis of each set of measuring result, the GDOP of positioning accuracy is $0.36 \mathrm{~m}$, which implies that the method can realize a high precision position of the distributed platforms.

\section{Summary}

This paper proposed a method for positioning combined two-dimensional angle measurement with ranging between high dynamic distributed platforms. Proposed a two-way microwave ranging method based on wideband positive and negative slope LFM signal which could suppress the Doppler interference effectively. Also proposed an angle measurement method with the phase ambiguity resolution by frequency estimation according to the characteristics of LFM signal. By analyzing the model and derivation, the feasibility of each methods are verified. The accuracy of ranging and positioning is verified by theoretical analysis and experiments.

\section{References}

[1]. D.J. Torrieri. Statistical theory of passive location systems. IEEE Transactions on Aerospace and Electronic Systems, 1984, 20(2):183-198.

[2]. Ho K C, Chan Y T, Solution and performance analysis of geolocation by TDOA [J]. IEEE Trans. on Aerospace and Electronic Systems, 1993, 29(4):1311-1322.

[3]. Wang L, Wei X, Wan J X, et al. A method of eliminating ambiguous solutions based on the TDOA algorithm [J]. Computer Engineering\&Science, 2006, 28(3): 74-75.

[4]. Wang Y C, Zhang L K, Position location using TDOA measurements in multisites [J]. Modern Radar, 2003, 25(2):1-4.

[5]. Yan F J, Gao X C, Zhu D Z. A study on the MTI cancellation method based on pulse-to- pulse alternation of modulation [J]. Radar and Confront. 2009, 4 (4):10-12.

[6]. Sakamoto Y, Nishio M. Orbit determination using radio interferometer of small-diameter antennas for LEO satellites [J]. IEEE Transactions on Aerospace and Electronic System, 2011, 47(3):2111 -2118.

[7]. Wu F W, Cheng T, Jia K X, et al. Algorithm of interferometer in direction finding based on virtual array transformation [J]. Modern Rardar. 2012, 34(3): 42-49.

[8]. Mao H, Yang J B, Liu P. The actuality and dev- eloppment of phase interferometer technology [J]. Electronic Warefare Technology, 2010, 25(6):1-6.

[9]. Musicki D. Koch W, Geolocation using TDOA and FDOA measurements[C]. Proc. of the 11th International Conference on Information Fusion, 2008:1-8.

[10]. Si W J, Chu P, Sun S H. Study on the methods of sloving direction finding ambiguity in very wideband[J].Journal of Projectiles, Rockets, Missiles and Guidance, 2009, 29(2): 45-48.

[11]. Jacobs E, Ralston E W. Ambiguity resolution in interferometry [J]. IEEE Transactions on Aerospace and Electronic System, 1981, 17(6): 766-780.

[12]. Chen T, Han Q Q, Liu L T, Lin J Q. Improved wide-band direction finding algorithm based on cross-spectra. 2014, 36(5):879-883.

[13]. Wang Y C, Zhang L K, Position location using TDOA measurements in multisites [J]. Modern Radar, 2003, 25(2):1-4. 